Research Article

\title{
Comparison of the Wiener and Kirchhoff Indices of Random Pentachains
}

\author{
Shouliu Wei $\mathbb{D}^{1}{ }^{1}$ Wai Chee Shiu, ${ }^{2,3}$ Xiaoling Ke, ${ }^{1}$ and Jianwu Huang ${ }^{1}$ \\ ${ }^{1}$ College of Mathematics and Data Science (Software College), Minjiang University, Fuzhou, Fujian 350108, China \\ ${ }^{2}$ College of Global Talents, Beijing Institute of Technology, 224 Zhuhai, Guangdong 519088, China \\ ${ }^{3}$ Department of Mathematics, The Chinese University of Hong Kong, Shatin, Hong Kong, China
}

Correspondence should be addressed to Shouliu Wei; wslwillow@126.com

Received 15 September 2021; Accepted 16 October 2021; Published 27 October 2021

Academic Editor: Elena Guardo

Copyright ( 92021 Shouliu Wei et al. This is an open access article distributed under the Creative Commons Attribution License, which permits unrestricted use, distribution, and reproduction in any medium, provided the original work is properly cited.

Let $G$ be a connected (molecule) graph. The Wiener index $W(G)$ and Kirchhoff index $K f(G)$ of $G$ are defined as the sum of distances and the resistance distances between all unordered pairs of vertices in $G$, respectively. In this paper, explicit formulae for the expected values of the Wiener and Kirchhoff indices of random pentachains are derived by the difference equation and recursive method. Based on these formulae, we then make comparisons between the expected values of the Wiener index and the Kirchhoff index in random pentachains and present the average values of the Wiener and Kirchhoff indices with respect to the set of all random pentachains with $n$ pentagons.

\section{Introduction}

All graphs considered in this paper are simple, undirected, and connected. All notations not defined in this paper can be found in [1]. Let $G$ be a graph with the vertex set $V(G)=$ $\left\{v_{1}, v_{2}, \ldots, v_{n}\right\}$ and edge set $E(G)$. The traditional distance $d\left(v_{r}, v_{s}\right)$ between vertices $v_{r}$ and $v_{s}$ is the number of edges of a shortest path connecting these vertices in G. In 1993, Klein and Randić [2] conceived the novel concept of resistance distance based on the electric resistance in an electrical network $N$ corresponding to the considered graph $G$, in which the resistance between any two adjacent nodes is $1 \mathrm{Ohm}$. Similar to the long recognized shortest path distance, the resistance distance is also intrinsic to the graph, not only with some nice purely mathematical and physical interpretations $[2,3]$, but also with a substantial potential for chemical applications. Denote by $r\left(v_{i}, v_{j}\right)$ the resistance distance between the vertices $v_{i}$ and $v_{j}$ in $G$, which is computed by Ohm's and Kirchhoff's laws in $N$.

For the resistance distance of a connected graph, a formula for computing it was presented by Klein and Randić [2] in 1993. We may compute the distance of two vertices in a connected graph $G$ of order $n$ from its Laplacian matrix. Precisely, let $L$ be the Laplacian matrix of $G$ and $J$ be the $n \times n$ matrix whose entries are 1. Let $X=(L+(1 / n) J)^{-1}$. Then, the resistance distance between vertices $i$ and $j$ of $G$ is

$$
r(i, j)=X_{i, i}+X_{j, j}-2 X_{i, j} .
$$

The Wiener index $W(G)[4,5]$ of a graph $G$ was first introduced by Wiener in the study of paraffin boiling points and a graph invariant based on distances in 1947. This is the oldest topological index related to molecular branching [6]. It is defined as the sum of distances between all unordered pairs of vertices in $G$, i.e.,

$$
W(G)=\frac{1}{2} \sum_{r=1}^{n} \sum_{s=1}^{n} d\left(v_{r}, v_{s}\right)=\frac{1}{2} \sum_{r=1}^{n} d\left(v_{r} \mid G\right),
$$

where $d\left(v_{i} \mid G\right)$ denotes the sum of distances between $v_{i}$ and all other vertices of $G$, defined by

$$
d\left(v_{i} \mid G\right)=\sum_{j=1}^{n} r\left(v_{i}, v_{j}\right) .
$$


For a survey of results and bibliography on the chemical applications and the mathematical literature of the Wiener index, see [7-15] and the references cited therein.

Similar to the Wiener index, the Kirchhoff index $K f(G)$ of a graph $G[2,16]$ is defined as

$$
K f(G)=\frac{1}{2} \sum_{i=1}^{n} \sum_{j=1}^{n} r\left(v_{i}, v_{j}\right)=\frac{1}{2} \sum_{i=1}^{n} r\left(v_{i} \mid G\right),
$$

where $r\left(v_{i} \mid G\right)$ denotes the sum of resistance distances between $v_{i}$ and all other vertices of $G$, defined by

$$
r\left(v_{i} \mid G\right)=\sum_{j=1}^{n} r\left(v_{i}, v_{j}\right) .
$$

Klein and Randić [2] proved that $r\left(v_{i}, v_{j}\right) \leq d\left(v_{i}, v_{j}\right)$ and $K f(G) \leq W(G)$ with equality if and only if $G$ is a tree. Like many topological indices, the Kirchhoff index based on resistance distance is also a graph invariant and molecular structure descriptor. Unfortunately, it is difficult to implement some algorithms [2, 17, 18] to compute resistant distance and Kirchhoff index in a graph from their computational complexity. Hence, it makes sense to find explicit closed-form formula for the Kirchhoff index. Up to now, the Kirchhoff index has been found to have noteworthy applications in chemistry, such as in assessing cyclicity of polycyclic structures including fullerenes, linear hexagonal chains, and some special molecular graphs such as distanceregular graphs and Möbius ladders [17-21]. Bonchev et al. in [16] considered the polymer science by using it and found that the Kirchhoff index in their approach is especially useful for defining the topological radius $R_{\text {top }}=\left(K f / n^{2}\right)$ of macromolecules containing cyclic fragments. Many mathematical properties have also been established (see [22-41] and the references cited therein).

Motivated by the works in [42-44], in this paper, we derive explicit formulae for the expected values of the Kirchhoff indices of random alpha-pentachain [43] and spiro-pentachain as shown in Figure 1 and Wiener index of random spiropentachain. Based on these formulae, we then make comparisons between the expected values of the Wiener index and the Kirchhoff index in random pentachains and present the average values of the Wiener and Kirchhoff indices with respect to the set of all random pentachains with $n$ pentagons. In fact, there also exist numerous research results for a general pentachain. For example, Xiao et al. [45] studied a connection between the Kekule structures of pentachains and the Hosoya index of caterpillar trees, and a general expression for calculating the bond incident degree indices of pentachains was established in [46]. Denote by $E[\Xi]$ the expected value of a random variable.

\section{Wiener and Kirchhoff Indices in Alpha- Pentachains}

An alpha-pentachain of length $n$ is obtained from a sequence of 5 cycles (pentagons) $C_{1}, C_{2}, \ldots, C_{n}$ by adding a bridge to each pair of consecutive 5 cycles, which is denoted by $P_{n}^{\alpha}$. The cycle $C_{i}$ will be called the $i$-th pentagon of $P_{n}^{\alpha}, 1 \leq i \leq n$.
Figure 2 shows the unique alpha-pentachains for $n=1,2$ and all the alpha-pentachains for $n=3$, and Figure 3 shows a general case, where $v_{n-1}$ is a vertex of $C_{n-1}$ in $P_{n-1}^{\alpha}$. Note that there are two ways to add a bridge between two consecutive cycles. So, $P_{n}^{\alpha}$ may not be unique when $n \geq 3$.

Consider $P_{n-1}^{\alpha}$ for $n \geq 3$. Let $C_{n-1}=x_{1} x_{2} x_{3} x_{4} x_{5} x_{1}$, and there is a bridge connecting $x_{1}$ and $v_{n-2}$ which is a vertex in $C_{n-2}$. There are two possible constructions of getting $P_{n}^{a}$ from $P_{n-1}^{\alpha}$ by the symmetry of the structure of an alpha-pentachain (see Figure 4). Precisely, let $P_{n}^{\alpha_{1}}$ and $P_{n}^{\alpha_{2}}$ be obtained by adding a bridge connecting a vertex of the extra pentagon $C_{n}$ with the vertices $x_{2}$ and $x_{3}$ of $C_{n-1}$, respectively (see Figure 4). We may assume that for getting a $P_{n}^{\alpha}$ from a fixed $P_{n-1}^{\alpha}$ is a random process, namely, the probability of getting $P_{n}^{\alpha_{1}}$ and $P_{n}^{\alpha_{2}}$ from a fixed $P_{n-1}^{\alpha}$ is $p$ and $1-p$, respectively.

We assume that the probability $p$ is constant and independent of $n$, i.e., the process described above is a zerothorder Markov process. After associating probabilities, such an alpha-pentachain is called a random alpha-pentachain and denoted by $P_{n ; p}^{\alpha}$. Gutman et al. $[47,48]$ considered the Wiener indices of random benzenoid chain graphs in 1990s. Chen and Zhang [49] obtained explicit analytical expressions for the expected value of the Wiener index and the number of perfect matchings in a random phenylene chain. Yang and Zhang [15] studied the Wiener indices of random polyphenyl chain graphs in 2012. Wei et al. [44, 50, 51] obtained simple formulae for the expected value of the Wiener indices of random polygonal chains. Wang et al. [43] studied the expected value of the Wiener indices of random alpha-pentachains.

Theorem 1 (see [43]). Let $P_{n ; p}^{\alpha}$ be a random alpha-pentachain. Then,

$$
E\left[W\left(P_{n ; p}^{\alpha}\right)\right]=\frac{5}{6}(15-5 p) n^{3}+\left(5+\frac{25}{2} p\right) n^{2}-\left(\frac{5}{2}+\frac{25}{3} p\right) n .
$$

In addition, Huang et al. [42] considered the problem about the Kirchhoff index in random alpha-pentachains. Wei and Ke [44] presented a simple formula for the expected value of Kirchhoff index in random generalized polyomino chain. For more details concerning random chain graphs, one may refer to [52-55] and the references cited therein. In this section, we will further consider the Kirchhoff index of random alpha-pentachain and have the following recurrence formula for computing it.

Lemma 1 (see [2]). Let y be a cut vertex of a connected graph $G$, and let $a$ and $b$ be vertices occurring in different components which arise upon deletion of $y$. Then,

$$
r(a, b)=r(a, y)+r(y, b) .
$$

Lemma 2. Let $P_{n}^{\alpha}$ be an alpha-pentachain of length $n$, where $n \geq 2$. Then,

$$
K f\left(P_{n}^{\alpha}\right)=K f\left(P_{n-1}^{\alpha}\right)+5 r\left(v_{n-1} \mid P_{n-1}^{\alpha}\right)+45 n-35 .
$$




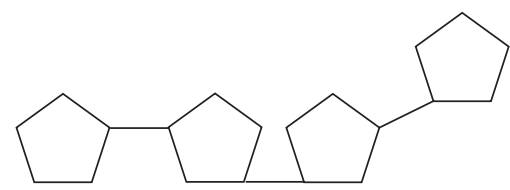

(a)

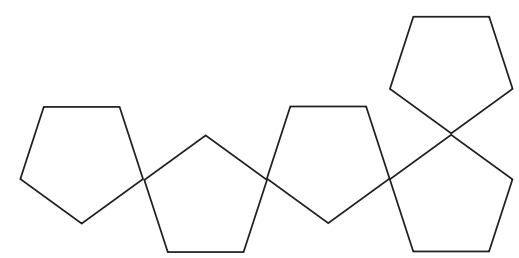

(b)

FIgURE 1: Two types of pentachains considered in this paper: (a) alpha-pentachain; (b) spiro-pentachain.

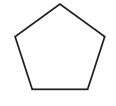

$P_{1}^{\alpha}$

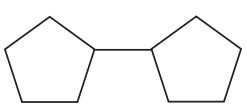

$P_{2}^{\alpha}$

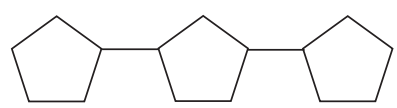

$P_{3}^{\alpha}$

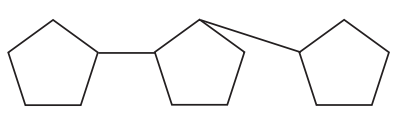

$P_{3}^{\alpha_{2}}$

Figure 2: The alpha-pentachains for $n=1,2,3$.

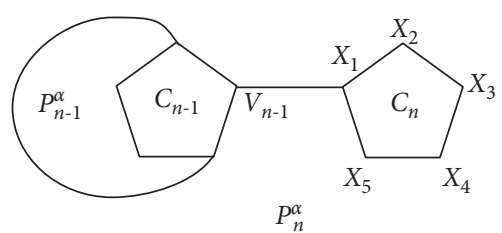

FIgURE 3: An alpha-pentachain $P_{n}^{\alpha}$ with $n$ pentagons.

Proof. Keeping the notation defined in Figure 3, note that by (1), the resistance distance matrix of the graph induced by $\left\{v_{n-1}, x_{1}, x_{2}, x_{3}, x_{4}, x_{5}\right\}$ is

$$
\left(\begin{array}{cccccc}
0 & 1 & \frac{9}{5} & \frac{11}{5} & \frac{11}{5} & \frac{9}{5} \\
1 & 0 & \frac{4}{5} & \frac{6}{5} & \frac{6}{5} & \frac{4}{5} \\
\frac{9}{5} & \frac{4}{5} & 0 & \frac{4}{5} & \frac{4}{5} & \frac{6}{5} \\
\frac{11}{5} & \frac{6}{5} & \frac{4}{5} & 0 & \frac{4}{5} & \frac{6}{5} \\
\frac{11}{5} & \frac{6}{5} & \frac{4}{5} & \frac{4}{5} & 0 & \frac{4}{5} \\
\frac{9}{5} & \frac{4}{5} & \frac{6}{5} & \frac{6}{5} & \frac{4}{5} & 0
\end{array}\right)
$$

By Lemma 1 and the matrix above, it is easy to check that

$$
r\left(x_{j}, v\right)= \begin{cases}r\left(v_{n-1}, v\right)+1, & \text { for } j=1, \\ r\left(v_{n-1}, v\right)+\frac{9}{5}, & \text { for } j=2,5, \\ r\left(v_{n-1}, v\right)+\frac{11}{5}, & \text { for } j=3,4,\end{cases}
$$

for any vertex $v$ of $P_{n-1}^{\alpha}$. Also, for a fixed vertex $j$, we have

$$
\sum_{i=1}^{5} r\left(x_{j}, x_{i}\right)=\frac{4}{5}+\frac{6}{5}+\frac{6}{5}+\frac{4}{5}=4,
$$

where $1 \leq j \leq 5$. Note that $P_{n-1}^{\alpha}$ has $5(n-1)$ vertices. Combining the above formulae, we obtain the following formulae:

$$
\begin{aligned}
& r\left(x_{1} \mid P_{n}^{\alpha}\right)=r\left(v_{n-1} \mid P_{n-1}^{\alpha}\right)+1 \times 5(n-1)+4=r\left(v_{n-1} \mid P_{n-1}^{\alpha}\right)+5 n-1, \\
& r\left(x_{2} \mid P_{n}^{\alpha}\right)=r\left(x_{5} \mid P_{n}^{\alpha}\right)=r\left(v_{n-1} \mid P_{n-1}^{\alpha}\right)+\frac{9}{5} \times 5(n-1)+4=r\left(v_{n-1} \mid P_{n-1}^{\alpha}\right)+9 n-5, \\
& r\left(x_{3} \mid P_{n}^{\alpha}\right)=r\left(x_{4} \mid P_{n}^{\alpha}\right)=r\left(v_{n-1} \mid P_{n-1}^{\alpha}\right)+\frac{11}{5} \times 5(n-1)+4=r\left(v_{n-1} \mid P_{n-1}^{\alpha}\right)+11 n-7 .
\end{aligned}
$$




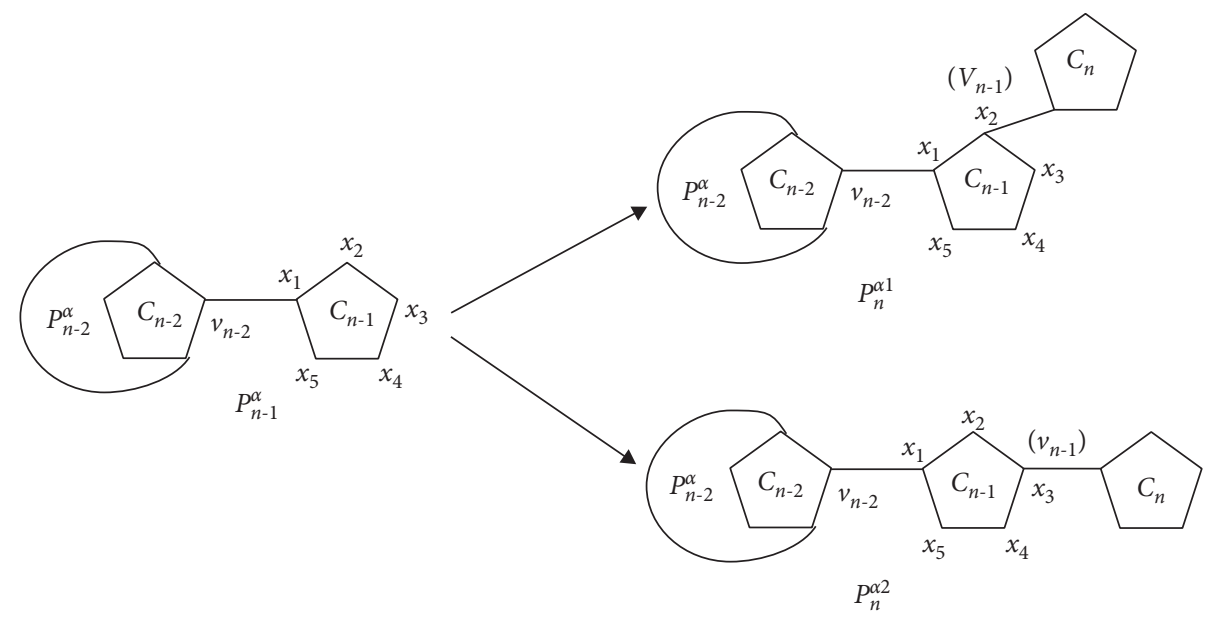

Figure 4: Two types of local arrangements in an alpha-pentachain.

Hence,

$$
\begin{aligned}
K f\left(P_{n}^{\alpha}\right)= & K f\left(P_{n-1}^{\alpha}\right)+5 r\left(v_{n-1} \mid P_{n-1}^{\alpha}\right)+45 n-25 \\
& -\frac{1}{2} \sum_{i=1}^{5} \sum_{j=1}^{5} r\left(x_{i}, x_{j}\right) \\
= & K f\left(P_{n-1}^{\alpha}\right)+5 r\left(v_{n-1} \mid P_{n-1}^{\alpha}\right)+45 n-35 .
\end{aligned}
$$

This completes the proof.

Note that both $K f\left(P_{n ; p}^{\alpha}\right)$ and $r\left(v_{n-1} \mid P_{n-1 ; p}^{\alpha}\right)$ are random variables. For convenience, let $R_{n-1}^{\alpha}=E\left[r\left(v_{n-1} \mid P_{n-1 ; p}^{\alpha}\right)\right]$ for $n \geq 2$. We will keep these notations throughout this section. In order to obtain the explicit expression of $E\left[K f\left(P_{n ; p}^{\alpha}\right)\right]$, we need to find the explicit expression of $R_{n-1}^{\alpha}$ from Lemma 2.

Lemma 3. Let $P_{n ; p}^{\alpha}$ be a random alpha-pentachain of length $n$, where $n \geq 2$. Then,

$$
R_{n-1}^{\alpha}=\left(\frac{11}{2}-p\right)(n-1)^{2}+\left(p-\frac{3}{2}\right)(n-1)
$$

Proof. When $n=2, P_{1 ; p}^{\alpha}$ is isomorphic to a pentagon. So,

$$
R_{1}^{\alpha}=E\left[r\left(v_{1} \mid P_{1 ; p}^{\alpha}\right)\right]=\frac{4}{5}+\frac{6}{5}+\frac{6}{5}+\frac{4}{5}=4 .
$$

When $n \geq 3$, there are two cases to be considered as shown in Figure 4. If $P_{n-1}^{\alpha} \longrightarrow P_{n}^{\alpha_{1}}$, then $v_{n-1}$ coincides with the vertex labeled $x_{2}$ or $x_{5}$. Consequently, $r\left(v_{n-1} \mid P_{n-1 ; p}^{\alpha}\right)$ is given by equation (12). If $P_{n-1}^{\alpha} \longrightarrow P_{n}^{\alpha_{2}}$, then $v_{n-1}$ coincides with the vertex labeled $x_{3}$ or $x_{4}$. Consequently, $r\left(v_{n-1} \mid P_{n-1 ; p}^{\alpha}\right)$ is given by equation (13). By symmetry, we may only consider $v_{n-1}$ which coincides with the vertex labeled $x_{i+1}$ for $i=1,2$.

Recall that the above two cases occur in random alphapentachains with probabilities $p$ and $1-p$. From equations $(2 \cdot i)(i=1,2)$, we obtain that

$$
R_{n-1}^{\alpha}=p\left\{E\left[r\left(v_{n-2} \mid P_{n-2 ; p}^{\alpha}\right)\right]+9(n-1)-5\right\}+(1-p)\left\{E\left[r\left(v_{n-2} \mid P_{n-2 ; p}^{\alpha}\right)\right]+11(n-1)-7\right\} .
$$

Note that $E\left[R_{n-1}^{\alpha}\right]=R_{n-1}^{\alpha}$. By applying the expectation operator to the above equation, we get

$$
\begin{aligned}
R_{n-1}^{\alpha} & =E\left[R_{n-1}^{\alpha}\right]=E\left[p\left(R_{n-2}^{\alpha}+9(n-1)-5\right)+(1-p)\left(R_{n-2}^{\alpha}+11(n-1)-7\right)\right] \\
& =p\left(R_{n-2}^{\alpha}+9(n-1)-5\right)+(1-p)\left(R_{n-2}^{\alpha}+11(n-1)-7\right) .
\end{aligned}
$$

That is,

$$
R_{n-1}^{\alpha}=R_{n-2}^{\alpha}+(11-2 p)(n-1)+(2 p-7), \quad \text { for } n \geq 3 .
$$

Note that equation (19) is a first-order nonhomogeneous linear difference equation with constant coefficients. It is clear that the general solution of the homogeneous part of equation (19) is $R^{\alpha}=c$, a constant.
Let $\quad R^{\alpha *}=(n-1)[a(n-1)+b]=a(n-1)^{2}+b(n-1)$ be a particular solution of equation (19). By substituting $R^{\alpha *}$ into equation (19), we have

$$
\begin{aligned}
& a(n-1)^{2}+b(n-1)-a(n-2)^{2}-b(n-2) \\
& =2 a(n-1)+b-a=(11-2 p)(n-1)+(2 p-7) .
\end{aligned}
$$

By comparing the coefficients of $n$ and the constant term, we have 


$$
\begin{aligned}
& a=\frac{11}{2}-p, \\
& b=p-\frac{3}{2} .
\end{aligned}
$$

Consequently, the general solution of equation (19) is $R_{n-1}^{\alpha}=R^{\alpha *}+R^{\alpha}=\left(\frac{11}{2}-p\right)(n-1)^{2}+\left(p-\frac{3}{2}\right)(n-1)+c$,

for $n \geq 2$.

By substituting the initial condition, we obtain that $c=0$. Hence, we get the lemma.

Theorem 2. Let $P_{n: p}^{\alpha}$ be a random alpha-pentachain of length $n$, where $n \geq 1$. Then,

$$
E\left[K f\left(P_{n ; p}^{\alpha}\right)\right]=\left(\frac{55}{6}-\frac{5}{3} p\right) n^{3}+(5 p+5) n^{2}-\left(\frac{10}{3} p+\frac{25}{6}\right) n \text {. }
$$

Proof. Since $P_{1}^{\alpha}$ is unique, $E_{1}$ is exactly the Kirchhoff index of $P_{1}^{\alpha}$, namely, $E_{1}=K f\left(P_{1}^{\alpha}\right)=10$. So, the theorem holds when $n=1$. When $n \geq 2$, by Lemmas 2 and 3 , we have

$$
\begin{aligned}
E_{n} & =E\left[K f\left(P_{n ; p}^{\alpha}\right)\right] \\
& =E\left[K f\left(P_{n-1 ; p}^{\alpha}\right)+5 r\left(v_{n-1} \mid P_{n-1 ; p}^{\alpha}\right)+45 n-35\right] \\
& =E_{n-1}+5 R_{n-1}^{\alpha}+45 n-35 \\
& =E_{n-1}+5\left[\left(\frac{11}{2}-p\right)(n-1)^{2}+\left(p-\frac{3}{2}\right)(n-1)\right]+45 n-35 \\
& =E_{n-1}+\left(\frac{55}{2}-5 p\right) n^{2}+\left(15 p-\frac{35}{2}\right) n-10 p .
\end{aligned}
$$

Clearly, $E=c$, a constant, is the general solution for the homogeneous part of equation (24).

Let $E^{*}=a_{0} n^{3}+a_{1} n^{2}+a_{2} n$ be a particular solution of equation (24). By substituting $E^{*}$ into equation (24), we have

$$
\begin{aligned}
& a_{0} n^{3}+a_{1} n^{2}+a_{2} n-\left[a_{0}(n-1)^{3}+a_{1}(n-1)^{2}+a_{2}(n-1)\right] \\
& =3 a_{0} n^{2}+\left(2 a_{1}-3 a_{0}\right) n+\left(a_{0}-a_{1}+a_{2}\right) \\
& =\left(\frac{55}{2}-5 p\right) n^{2}+\left(15 p-\frac{35}{2}\right) n-10 p .
\end{aligned}
$$

By comparing the coefficients of $n^{i}$ term for $0 \leq i \leq 2$, we have

$$
\begin{aligned}
& a_{0}=\frac{55}{6}-\frac{5}{3} p, \\
& a_{1}=5 p+5, \\
& a_{2}=-\frac{10}{3} p-\frac{25}{6} .
\end{aligned}
$$

Combining the result and the initial condition, we get that the general solution of equation (24) is

$$
E_{n}=E^{*}+E=\left(\frac{55}{6}-\frac{5}{3} p\right) n^{3}+(5 p+5) n^{2}-\left(\frac{10}{3} p+\frac{25}{6}\right) n .
$$

Theorems 1 and 2 show that both the function $E\left[W\left(P_{n ; p}^{\alpha}\right)\right]$ and $E\left[K f\left(P_{n ; p}^{\alpha}\right)\right]$ are asymptotic to a cubic in $n$ and linear in $p$. In particular, it is easy to obtain the Wiener and Kirchhoff indices of two special alpha-pentachains $P_{n ; 1}^{\alpha}$ and $P_{n: 0}^{\alpha}$ when setting $p=1$ and $p=0$ from Theorems 1 and 2.

Corollary 1. The Wiener indices of the alpha-pentachains $P_{n ; 1}^{\alpha}$ and $P_{n ; 0}^{\alpha}$ are

$$
\begin{aligned}
& W\left(P_{n ; 1}^{\alpha}\right)=\frac{25}{3} n^{3}+\frac{35}{2} n^{2}-\frac{65}{6} n, \\
& W\left(P_{n ; 0}^{\alpha}\right)=\frac{25}{2} n^{3}+5 n^{2}-\frac{5}{2} n .
\end{aligned}
$$

Corollary 2. The Kirchhoff indices of the alpha-pentachains $P_{n ; 1}^{\alpha}$ and $P_{n ; 0}^{\alpha}$ are

$$
\begin{aligned}
& K f\left(P_{n ; 1}^{\alpha}\right)=\frac{15}{2} n^{3}+10 n^{2}-\frac{15}{2} n, \\
& K f\left(P_{n ; 0}^{\alpha}\right)=\frac{55}{6} n^{3}+5 n^{2}-\frac{25}{6} n .
\end{aligned}
$$

Klein and Randić [2] proved that $K f(G) \leq W(G)$ with equality if and only if $G$ is a tree. Combining Theorem 1 with Theorem 2, we can also verify that $E\left[K f\left(P_{n ; p}^{\alpha}\right)\right]<E\left[W\left(P_{n ; p}^{\alpha}\right)\right]$.

Theorem 3. Let $P_{n ; p}^{\alpha}$ be a random alpha-pentachain. Then,

$$
E\left[K f\left(P_{n ; p}^{\alpha}\right)\right]<E\left[W\left(P_{n ; p}^{\alpha}\right)\right] .
$$

Proof. By Theorems 1 and 2, we have

$$
\begin{aligned}
6 E\left[W\left(P_{n ; p}^{\alpha}\right)\right]-6 E\left[K f\left(P_{n ; p}^{\alpha}\right)\right] & =(20-20 p) n^{3}+45 p n^{2}+(10-30 p) n \\
& =(20-20 p) n^{3}+n[(45 n-30) p+10] .
\end{aligned}
$$


Since $0 \leq p \leq 1, \quad(20-20 p) n^{3}+n[(45 n-30) p+10]>0$, which implies that

$$
E\left[K f\left(P_{n ; p}^{\alpha}\right)\right]<E\left[W\left(P_{n ; p}^{\alpha}\right)\right] .
$$

Hence, this completes the proof.

\section{Wiener and Kirchhoff Indices in Spiro- Pentachains}

The notation not defined in this section can be found in Section 2. A spiro-pentachain of length $n$, denoted by $P_{n}^{s}$, can be obtained from an alpha-pentachain $P_{n}^{\alpha}$ by contracting each bridge between each pair of consecutive pentagons in $P_{n}^{\alpha}$. Figure 5 shows a general spiro-pentachain, where $v_{n-1}$ is a vertex of $C_{n-1}$ in $P_{n-1}^{s}$. Clearly, $P_{n}^{s}$ may not be unique when $n \geq 3$. Corresponding to the structure of alpha-pentachain $P_{n}^{\alpha}$, the spiro-pentachain $P_{n}^{s}$ has also two types of local arrangements which are denoted by $P_{n}^{s_{1}}$ and $P_{n}^{s_{2}}$ (see Figure 6). We may also assume that for getting $P_{n}^{s}$ from fixed $P_{n-1}^{s}$ is a random process, namely, the probability of getting $P_{n}^{s_{1}}$ and $P_{n}^{s_{2}}$ from fixed $P_{n-1}^{s}$ is $p$ and $1-p$, respectively.

We assume that the probability $p$ is constant and independent of $n$, i.e., the process described above is a zerothorder Markov process. After associating probabilities, such a spiro-pentachain is called a random spiro-pentachain and denoted by $P_{n ; p}^{s}$. In this section, we will consider the Wiener and Kirchhoff indices of random spiro-pentachain.

Lemma 4. Let $P_{n}^{s}$ be a spiro-pentachain of length $n$, where $n \geq 2$. Then, the Wiener index $W\left(P_{n}^{s}\right)$ of $P_{n}^{s}$ is

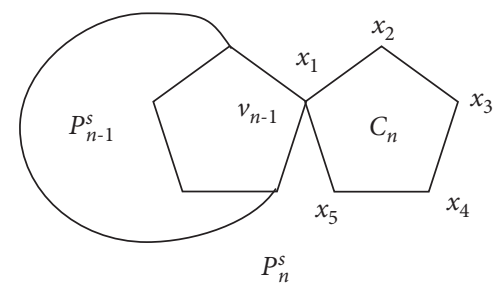

FIgURE 5: A spiro-pentachain $P_{n}^{s}$ with $n$ pentagons.

$$
W\left(P_{n-1}^{s}\right)+4 d\left(v_{n-1} \mid P_{n-1}^{s}\right)+24 n-9
$$

Proof. Keeping the notation defined in Figure 5, it is easy to check that

$$
d\left(x_{j}, v\right)= \begin{cases}d\left(v_{n-1}, v\right), & \text { for } j=1, \\ d\left(v_{n-1}, v\right)+1, & \text { for } j=2,5, \\ d\left(v_{n-1}, v\right)+2, & \text { for } j=3,4,\end{cases}
$$

for any vertex $v$ of $P_{n-1}^{s}$. Also, for a fixed vertex $j$, we have

$$
\sum_{i=1}^{5} d\left(x_{j}, x_{i}\right)=1+1+2+2=6,
$$

where $1 \leq j \leq 5$. Note that $P_{n-1}^{s}$ has $5(n-1)-(n-2)=4 n-$ 3 vertices and $x_{1}$ coincides with $v_{n-1}$ in $P_{n}^{s}$. Combining the above formulae, we obtain the following formulae:

$$
\begin{aligned}
& \text { Hence, } \\
& \begin{aligned}
W\left(P_{n}^{s}\right)= & W\left(P_{n-1}^{s}\right)+6+4 d\left(v_{n-1} \mid P_{n-1}^{s}\right)+24 n \\
& -\frac{1}{2} \sum_{i=1}^{5} \sum_{j=1}^{5} d\left(x_{i}, x_{j}\right) \\
= & W\left(P_{n-1}^{s}\right)+4 d\left(v_{n-1} \mid P_{n-1}^{s}\right)+24 n-9 .
\end{aligned}
\end{aligned}
$$$$
d\left(x_{1} \mid P_{n}^{s}\right)=d\left(v_{n-1} \mid P_{n}^{s}\right)=d\left(v_{n-1} \mid P_{n-1}^{s}\right)+6,
$$$$
d\left(x_{2} \mid P_{n}^{s}\right)=d\left(x_{5} \mid P_{n}^{s}\right)=d\left(v_{n-1} \mid P_{n-1}^{s}\right)+4(n-1)+6=d\left(v_{n-1} \mid P_{n-1}^{s}\right)+4 n+2 \text {, }
$$$$
d\left(x_{3} \mid P_{n}^{s}\right)=d\left(x_{4} \mid P_{n}^{s}\right)=d\left(v_{n-1} \mid P_{n-1}^{s}\right)+2 \times 4(n-1)+6=d\left(v_{n-1} \mid P_{n-1}^{s}\right)+8 n-2 .
$$$$
D_{n-1}^{s}=(4-2 p)(n-1)^{2}+(2 p+2)(n-1) .
$$

This completes the proof.

Note that both $W\left(P_{n ; p}^{s}\right)$ and $r\left(v_{n-1} \mid P_{n-1 ; p}^{s}\right)$ are also random variables. In order to obtain the explicit expression of $E\left[W\left(P_{n: p}^{s}\right)\right]$, we need to find the explicit expression of $D_{n-1}^{s} \triangleq E\left[d\left(v_{n-1} \mid P_{n-1}^{s}\right)\right]$ for $n \geq 2$ from Lemma 4 .

Lemma 5. Let $P_{n ; p}^{s}$ be a random spiro-pentachain of length $n$, where $n \geq 2$. Then,
Proof. When $n=2, P_{1 ; p}^{s}$ is isomorphic to a pentagon. So,

$$
D_{1}^{s}=E\left[d\left(v_{1} \mid P_{1 ; p}^{s}\right)\right]=6
$$

When $n \geq 3$, there are two cases to be considered as shown in Figure 6. If $P_{n-1}^{s} \longrightarrow P_{n}^{s_{1}}$, then $v_{n-1}$ coincides with the vertex labeled $x_{2}$ or $x_{5}$. Consequently, $d\left(v_{n-1} \mid P_{n-1 ; p}^{s}\right)$ is given by equation (36). If $P_{n-1}^{s} \longrightarrow P_{n}^{s_{2}}$, then $v_{n-1}$ coincides with the vertex labeled $x_{3}$ or $x_{4}$. Consequently, $d\left(v_{n-1} \mid P_{n-1 ; p}^{s}\right)$ is given by equation (37). By symmetry, we may only consider $v_{n-1}$ which coincides with the vertex labeled $x_{i+1}$ for $i=1,2$.

Recall that the above two cases occur in random spiropentachains with probabilities $p$ and $1-p$. From equations $(3 \cdot i)(i=1,2)$, we obtain that 


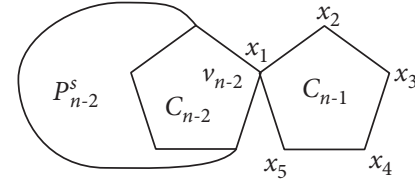

$P_{n-1}^{s}$

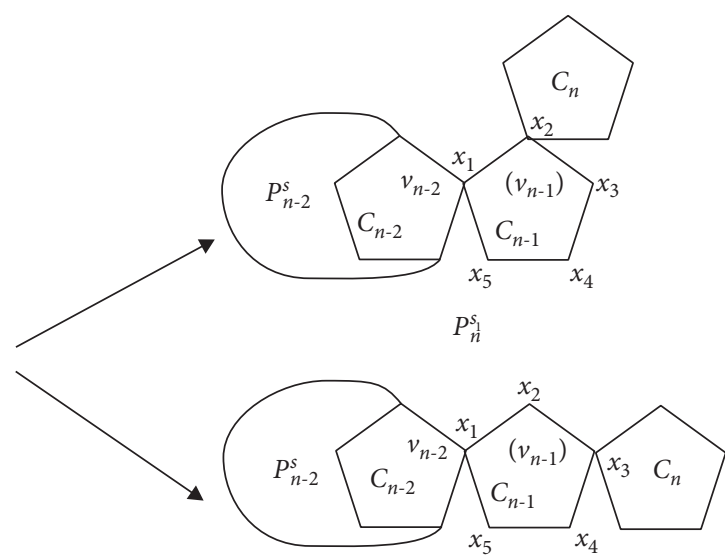

$P_{n}^{s_{2}}$

Figure 6: Two types of local arrangements in a spiro-pentachain.

$$
D_{n-1}^{s}=p\left\{E\left[d\left(v_{n-2} \mid P_{n-2 ; p}^{s}\right)\right]+4(n-1)+2\right\}+(1-p)\left\{E\left[d\left(v_{n-2} \mid P_{n-2 ; p}^{s}\right)\right]+8(n-1)-2\right\} .
$$

Note that $E\left[D_{n-1}^{s}\right]=D_{n-1}^{s}$. By applying the expectation operator to the above equation, we get

$$
\begin{aligned}
D_{n-1}^{s} & =E\left[D_{n-1}^{s}\right]=E\left[p\left(D_{n-2}^{s}+4(n-1)+2\right)+(1-p)\left(D_{n-2}^{s}+8(n-1)-2\right)\right] \\
& =p\left[D_{n-2}^{s}+4(n-1)+2\right]+(1-p)\left[D_{n-2}^{s}+8(n-1)-2\right] .
\end{aligned}
$$

That is,

$D_{n-1}^{s}=D_{n-2}^{s}+(8-4 p)(n-1)+(4 p-2), \quad$ for $n \geq 2$.

Using recurrence relation (43) and the initial condition, we have

$$
D_{n-1}^{s}=(4-2 p)(n-1)^{2}+(2 p+2)(n-1) .
$$

Hence, we get the lemma.

Theorem 4. Let $P_{n ; p}^{s}$ be a random spiro-pentachain of length $n$, where $n \geq 1$. Then,

$$
E\left[W\left(P_{n ; p}^{s}\right)\right]=\left(\frac{16}{3}-\frac{8}{3} p\right) n^{3}+(8 p+8) n^{2}+\left(\frac{5}{3}-\frac{16}{3} p\right) n .
$$

Proof. For convenience, let $E_{n}^{w}=E\left[W\left(P_{n ; p}^{s}\right)\right]$. Since $P_{1}^{s}$ is unique, $E_{1}^{w}$ is exactly the Wiener index of $P_{1}^{s}$, namely, $E_{1}^{w}=W\left(P_{1}^{s}\right)=15$. So, the theorem holds when $n=1$. When $n \geq 2$, by Lemmas 4 and 5 , we have

$$
\begin{aligned}
E_{n}^{w} & =E\left[W\left(P_{n ; p}^{s}\right)\right] \\
& =E\left[W\left(P_{n-1 ; p}^{s}\right)+4 d\left(v_{n-1} \mid P_{n-1 ; p}^{s}\right)+24 n-9\right] \\
& =E_{n-1}^{w}+4 D_{n-1}^{s}+24 n-9 \\
& =E_{n-1}^{w}+4\left[(4-2 p)(n-1)^{2}+(2 p+2)(n-1)\right]+24 n-9 \\
& =E_{n-1}^{w}+(16-8 p) n^{2}+24 p n-(1+16 p) .
\end{aligned}
$$

Using recurrence relation (46) and the initial condition, we have

$$
E\left[W\left(P_{n ; p}^{s}\right)\right]=\left(\frac{16}{3}-\frac{8}{3} p\right) n^{3}+(8 p+8) n^{2}+\left(\frac{5}{3}-\frac{16}{3} p\right) n
$$

We now give the formula for the expected value of the Kirchhoff index of a random spiro-pentachain.

Lemma 6. Let $P_{n}^{s}$ be a spiro-pentachain of length $n$, where $n \geq 2$. Then,

$$
K f\left(P_{n}^{s}\right)=K f\left(P_{n-1}^{s}\right)+4 r\left(v_{n-1} \mid P_{n-1}^{s}\right)+16 n-6 .
$$


Proof. Keeping the notation defined in Figure 5, note that the resistance distance matrix of the graph induced by $\left\{x_{1}, x_{2}, x_{3}, x_{4}, x_{5}\right\}$ is

$$
\left(\begin{array}{lllll}
0 & \frac{4}{5} & \frac{6}{5} & \frac{6}{5} & \frac{4}{5} \\
\frac{4}{5} & 0 & \frac{6}{5} & \frac{6}{5} & \frac{4}{5} \\
\frac{6}{5} & \frac{4}{5} & 0 & \frac{4}{5} & \frac{6}{5} \\
\frac{6}{5} & \frac{6}{5} & \frac{4}{5} & 0 & \frac{4}{5} \\
\frac{4}{5} & \frac{6}{5} & \frac{6}{5} & \frac{4}{5} & 0
\end{array}\right),
$$

by (1). By Lemma 1 and the matrix above, it is easy to check that

$$
r\left(x_{j}, v\right)= \begin{cases}r\left(v_{n-1}, v\right), & \text { for } j=1, \\ r\left(v_{n-1}, v\right)+\frac{4}{5}, & \text { for } j=2,5, \\ r\left(v_{n-1}, v\right)+\frac{6}{5}, & \text { for } j=3,4,\end{cases}
$$

for any vertex $v$ in $P_{n-1}^{s}$. From the proof of Lemma 2, we have

$$
\sum_{i=1}^{5} r\left(x_{j}, x_{i}\right)=4
$$

for a fixed $j, 1 \leq j \leq 5$. Note that $P_{n-1}^{s}$ has $5(n-1)-(n-2)=$ $4 n-3$ vertices and $x_{1}$ coincides with $v_{n-1}$ in $P_{n}^{s}$. Combining the formulae above, we obtain the following formulae:

$$
\begin{aligned}
r\left(x_{1} \mid P_{n}^{s}\right) & =r\left(v_{n-1} \mid P_{n}^{s}\right)=r\left(v_{n-1} \mid P_{n-1}^{s}\right)+4 \\
r\left(x_{2} \mid P_{n}^{s}\right) & =r\left(x_{5} \mid P_{n}^{s}\right)=r\left(v_{n-1} \mid P_{n-1}^{s}\right)+\frac{4}{5} \times 4(n-1)+4 \\
& =r\left(v_{n-1} \mid P_{n-1}^{s}\right)+\frac{4}{5}(4 n+1), \\
r\left(x_{3} \mid P_{n}^{s}\right) & =r\left(x_{4} \mid P_{n}^{s}\right)=r\left(v_{n-1} \mid P_{n-1}^{s}\right)+\frac{6}{5} \times 4(n-1)+4 \\
& =r\left(v_{n-1} \mid P_{n-1}^{s}\right)+\frac{4}{5}(6 n-1) .
\end{aligned}
$$

Hence,

$$
\begin{aligned}
K f\left(P_{n}^{s}\right)= & K f\left(P_{n-1}^{s}\right)+4+\left[r\left(v_{n-1} \mid P_{n-1}^{s}\right)+\frac{4}{5}(4 n+1)\right] \times 2 \\
& +\left[r\left(v_{n-1} \mid P_{n-1}^{s}\right)+\frac{4}{5}(6 n-1)\right] \times 2-\frac{1}{2} \sum_{i=1}^{5} \sum_{j=1}^{5} r\left(x_{i}, x_{j}\right) \\
= & K f\left(P_{n-1}^{s}\right)+4 r\left(v_{n-1} \mid P_{n-1}^{s}\right)+16 n-6 .
\end{aligned}
$$

This completes the proof.

Lemma 7. Let $P_{n ; p}^{s}$ be a random spiro-pentachain of length $n$, where $n \geq 2$. Then,

$$
R_{n-1}^{s}=\frac{4}{5}(3-p)(n-1)^{2}+\frac{4}{5}(2+p)(n-1),
$$

where $R_{n-1}^{s}=E\left[r\left(v_{n-1} \mid P_{n-1 ; p}^{s}\right)\right]$.

Proof. When $n=2$,

$$
R_{1}^{s}=E\left[r\left(v_{1} \mid P_{1 ; p}^{s}\right)\right]=\frac{4}{5}+\frac{6}{5}+\frac{6}{5}+\frac{4}{5}=4 .
$$

When $n \geq 3$, there are two cases to be considered as shown in Figure 6. If $P_{n-1}^{s} \longrightarrow P_{n}^{s_{1}}$, then $v_{n-1}$ coincides with the vertex labeled $x_{2}$ or $x_{5}$. Consequently, $r\left(v_{n-1} \mid P_{n-1 ; p}^{s}\right)$ is given by equation (52). If $P_{n-1}^{s} \longrightarrow P_{n}^{s_{2}}$, then $v_{n-1}$ coincides with the vertex labeled $x_{3}$ or $x_{4}$. Consequently, $r\left(v_{n-1} \mid P_{n-1 ; p}^{s}\right)$ is given by equation (53). By symmetry, we may only consider $v_{n-1}$ which coincides with the vertex labeled $x_{i+1}$ for $i=1,2$.

Recall that the above two cases occur in random spiropentachains with probabilities $p$ and $1-p$. From equations $(3 \cdot i)(i=5,6)$, we obtain that

$$
R_{n-1}^{s}=E\left\{p\left[r\left(v_{n-2} \mid P_{n-2 ; p}^{s}\right)+\frac{4}{5}[4(n-1)+1]\right]+(1-p)\left[r\left(v_{n-2} \mid P_{n-2 ; p}^{s}\right)+\frac{4}{5}[6(n-1)-1]\right]\right\} .
$$


By applying the expectation operator to the above equation, we get

$$
R_{n-1}^{s}=R_{n-2}^{s}+\frac{8}{5}(3-p)(n-1)+\frac{4}{5}(2 p-1), \quad \text { for } n \geq 2 .
$$

Using recurrence relation (58) and the initial condition, we have

$$
R_{n-1}^{s}=\frac{4}{5}(3-p)(n-1)^{2}+\frac{4}{5}(2+p)(n-1) .
$$

Hence, we get the lemma.

Similar to the proof in Theorem 2, now we have the following result by Lemmas 6 and 7 .

Theorem 5. Let $P_{n ; p}^{s}$ be a random spiro-pentachain of length $n$, where $n \geq 1$. Then,

$$
E\left[K f\left(P_{n ; p}^{s}\right)\right]=\frac{16}{15}(3-p) n^{3}+\frac{16}{5}(2+p) n^{2}+\frac{2}{15}(3-16 p) n .
$$

Similarly, Theorems 4 and 5 show that both the functions $E\left[W\left(P_{n ; p}^{s}\right)\right]$ and $E\left[K f\left(P_{n: p}^{s}\right)\right]$ are asymptotic to a cubic in $n$ and linear in $p$. Then, we easily get the Wiener and Kirchhoff indices of two special spiro-pentachains $P_{n ; 1}^{s}$ and $P_{n ; 0}^{s}$ when setting $p=1$ and $p=0$ from Theorems 4 and 5 .

Corollary 3. The Wiener indices of the spiro-pentachains $P_{n: 1}^{s}$ and $P_{n: 0}^{s}$ are

$$
\begin{aligned}
& W\left(P_{n ; 1}^{s}\right)=\frac{8}{3} n^{3}+16 n^{2}-\frac{11}{3} n, \\
& W\left(P_{n ; 0}^{s}\right)=\frac{16}{3} n^{3}+8 n^{2}+\frac{5}{3} n .
\end{aligned}
$$

Corollary 4. The Kirchhoff indices of the spiro-pentachains $P_{n ; 1}^{s}$ and $P_{n: 0}^{s}$ are

$$
\begin{aligned}
& K f\left(P_{n ; 1}^{s}\right)=\frac{32}{15} n^{3}+\frac{48}{5} n^{2}-\frac{26}{15} n, \\
& K f\left(P_{n ; 0}^{s}\right)=\frac{48}{15} n^{3}+\frac{32}{5} n^{2}+\frac{2}{5} n .
\end{aligned}
$$

By Theorems 4 and 5, similar to Theorem 3, we also have the following result.

Theorem 6. Let $P_{n ; p}^{s}$ be a random spiro-pentachain. Then,

$$
E\left[K f\left(P_{n ; p}^{s}\right)\right]<E\left[W\left(P_{n ; p}^{s}\right)\right] .
$$

\section{The Average Values of the Kirchhoff and Wiener Indices in Random Pentachains}

In this section, we present the average values of the Kirchhoff and Wiener indices in random pentachains with respect to the set of all alpha-pentachains and all spiro-pentachains with $n$ pentagons. Let $\mathscr{P}_{n}^{\alpha}$ and $\mathscr{P}_{n}^{s}$ be the sets of all alpha-pentachains and all spiro-pentachains with $n$ pentagons. The average values of Wiener indices of $\mathscr{P}_{n}^{\alpha}$ and $\mathscr{P}_{n}^{s}$ are defined by

$$
\begin{aligned}
W_{\text {avr }}\left(\mathscr{P}_{n}^{\alpha}\right) & =\frac{1}{\left|\mathscr{P}_{n}^{\alpha}\right|} \sum_{G \in \mathscr{P}_{n}^{s}} W(G), \\
W_{\text {avr }}\left(\mathscr{P}_{n}^{s}\right) & =\frac{1}{\left|\mathscr{P}_{n}^{s}\right|} \sum_{G \in \mathscr{P}_{n}^{s}} W(G),
\end{aligned}
$$

respectively. Similarly, the average values of Kirchhoff indices of $\mathscr{P}_{n}^{\alpha}$ and $\mathscr{P}_{n}^{s}$ are defined by

$$
\begin{aligned}
& K f_{\text {avr }}\left(\mathscr{P}_{n}^{\alpha}\right)=\frac{1}{\left|\mathscr{P}_{n}^{\alpha}\right|} \sum_{G \in \mathscr{P}_{n}^{s}} K f(G), \\
& K f_{\text {avr }}\left(\mathscr{P}_{n}^{s}\right)=\frac{1}{\left|\mathscr{P}_{n}^{s}\right|} \sum_{G \in \mathscr{P}_{n}^{s}} K f(G),
\end{aligned}
$$

respectively.

Actually, these are the population means of the Wiener and Kirchhoff indices of all elements in $\mathscr{P}_{n}^{\alpha}$ or $\mathscr{P}_{n}^{s}$. Since every element occurring in $\mathscr{P}_{n}^{\alpha}$ or $\mathscr{P}_{n}^{s}$ has the same probability, we have $p=1-p$. Thus, we may apply Theorems $1,2,4$, and 5 by putting $p=1-p=(1 / 2)$ and obtain the following results.

Theorem 7. The average values of the Wiener indices with respect to $\mathscr{P}_{n}^{s}$ and $\mathscr{P}_{n}^{\alpha}$ are

$$
\begin{aligned}
& W_{\text {avr }}\left(\mathscr{P}_{n}^{\alpha}\right)=\frac{125}{12} n^{3}+\frac{45}{4} n^{2}-\frac{20}{3} n, \\
& W_{\text {avr }}\left(\mathscr{P}_{n}^{s}\right)=4 n^{3}+12 n^{2}-n .
\end{aligned}
$$

Theorem 8. The average values of the Kirchhoff indices with respect to $\mathscr{P}_{n}^{s}$ and $\mathscr{P}_{n}^{\alpha}$ are

$$
\begin{aligned}
& K f_{\text {avr }}\left(\mathscr{P}_{n}^{\alpha}\right)=\frac{25}{3} n^{3}+\frac{15}{2} n^{2}-\frac{35}{6} n, \\
& K f_{\text {avr }}\left(\mathscr{P}_{n}^{s}\right)=4 n^{3}+12 n^{2}-n .
\end{aligned}
$$

It is not difficult to note that the average values of the Wiener indices with respect to $\left\{P_{n ; 1}^{\alpha}, P_{n ; 0}^{\alpha}\right\}$ and $\left\{P_{n ; 1}^{s}, P_{n ; 0}^{s}\right\}$ are

$$
\begin{aligned}
& \frac{W\left(P_{n ; 1}^{\alpha}\right)+W\left(P_{n ; 0}^{\alpha}\right)}{2}=\frac{125}{12} n^{3}+\frac{45}{4} n^{2}-\frac{20}{3} n, \\
& \frac{W\left(P_{n ; 1}^{s}\right)+W\left(P_{n ; 0}^{s}\right)}{2}=\frac{8}{3} n^{3}+8 n^{2}-\frac{2}{3} n,
\end{aligned}
$$

from Corollaries 1 and 3 and Theorem 8 . We also can find that the average values of the Kirchhoff indices with respect to $\left\{P_{n ; 1}^{\alpha}, P_{n ; 0}^{\alpha}\right\}$ and $\left\{P_{n ; 1}^{s}, P_{n ; 0}^{s}\right\}$ are 


$$
\begin{aligned}
& \frac{K f\left(P_{n ; 1}^{\alpha}\right)+K f\left(P_{n ; 0}^{\alpha}\right)}{2}=\frac{25}{3} n^{3}+\frac{15}{2} n^{2}-\frac{35}{6} n, \\
& \frac{K f\left(P_{n ; 1}^{s}\right)+K f\left(P_{n ; 0}^{s}\right)}{2}=\frac{8}{3} n^{3}+8 n^{2}-\frac{2}{3} n,
\end{aligned}
$$

from Corollaries 2 and 4 and Theorem 8. The interesting results show that both the average values of the Wiener indices and the average values of the Kirchhoff indices with respect to $\mathscr{P}_{n}^{\alpha}$ and $\mathscr{P}_{n}^{s}$ are exactly equal to the average value of the Wiener and Kirchhoff indices with respect to $\left\{P_{n ; 1}^{\alpha}, P_{n ; 0}^{\alpha}\right\}$ and $\left\{P_{n ; 1}^{s}, P_{n ; 0}^{s}\right\}$, respectively.

\section{Data Availability}

No data were used to support this study.

\section{Conflicts of Interest}

The authors declare that they have no potential conflicts of interest.

\section{Acknowledgments}

This work was partially finished while the first author visited Department of Mathematics, Hong Kong Baptist University. This work was supported by the National Natural Science Foundation of China (no. 12061007) and Natural Science Foundation of Fujian Province (no. 2020J01844).

\section{References}

[1] J. A. Bondy and U. S. R. Murty, Graph Theory with Applications, MacMillan, New York, NY, USA, 1976.

[2] D. J. Klein and M. Randić, "Resistance distance," Journal of Mathematical Chemistry, vol. 12, no. 1, pp. 81-95, 1993.

[3] D. J. Klein, "Graph geometry, graph metrics, and Wiener," MATCH Communications in Mathematical and in Computer Chemistry, vol. 35, pp. 7-27, 1997.

[4] H. Hosoya, "Topological index. A newly proposed quantity characterizing the topological nature of structural isomers of saturated hydrocarbons," Bulletin of the Chemical Society of Japan, vol. 44, no. 9, pp. 2332-2339, 1971.

[5] H. Wiener, "Structural determination of paraffin boiling points," Journal of the American Chemical Society, vol. 69, no. 1, pp. 17-20, 1947.

[6] R. Todeschini and V. Consonni, Handbook of Molecular Descriptors, Wiley-VCH, Hoboken, NJ, USA, 2000.

[7] H. Bian and F. Zhang, "Tree-like polyphenyl systems with extremal Wiener indices," MATCH Communications in Mathematical and in Computer Chemistry, vol. 61, pp. 631642, 2009.

[8] A. A. Dobrynin, R. Entringer, and I. Gutman, "Wiener index of trees: theory and applications," Acta Applicandae Mathematicae, vol. 66, no. 3, pp. 211-249, 2001.

[9] A. A. Dobrynin, I. Gutman, S. Žigert, and P. Žigert, "Wiener index of hexagonal systems," Acta Applicandae Mathematicae, vol. 72, no. 3, pp. 247-294, 2002.

[10] W. C. Shiu and P. C. B. Lam, "The Wiener number of the hexagonal net," Discrete Applied Mathematics, vol. 73, no. 2, pp. 101-111, 1997.
[11] W. C. Shiu and P. C. B. Lam, "Wiener number of pericondensed benzenoidmolecule systems," Congressus Numerantium, vol. 126, pp. 113-124, 1997.

[12] W. C. Shiu, C. S. Tong, and P. C. B. Lam, "Wiener number of hexagonal jagged-rectangles," Discrete Applied Mathematics, vol. 80, no. 1, pp. 83-96, 1997.

[13] W. C. Shiu, P. C. B. Lam, and I. Gutman, "Wiener number of hexagonal bitrapeziums and trapeziums," Bulletin Classe des Sciences Mathematiques et Natturalles, vol. 114, pp. 9-25, 1997.

[14] W. C. Shiu, P. C. B. Lam, and K. K. Poon, "On Wiener numbers of polygonal nets," Discrete Applied Mathematics, vol. 122, no. 1-3, pp. 251-261, 2002.

[15] W. Yang and F. Zhang, "Wiener index in random polyphenyl chains," MATCH Communications in Mathematical and in Computer Chemistry, vol. 68, pp. 371-376, 2012.

[16] D. Bonchev, A. T. Balaban, X. Liu, and D. J. Klein, "Molecular cyclicity and centricity of polycyclic graphs. I. Cyclicity based on resistance distances or reciprocal distances," International Journal of Quantum Chemistry, vol. 50, no. 1, pp. 1-20, 1994.

[17] D. Babić, D. J. Klein, I. Lukovits, S. Nikolić, and N. Trinajstić, "Resistance distance matrix: a com- putational algorithm and its application," International Journal of Quantum Chemistry, vol. 90, pp. 166-176, 2002.

[18] J. L. Palacios, "Closed-form formulas for Kirchhoff index," International Journal of Quantum Chemistry, vol. 81, no. 2, pp. 135-140, 2001.

[19] E. Estrada and N. Hatano, "Topological atomic displacements, Kirchhoff and Wiener indices of molecules," Chemical Physics Letters, vol. 486, no. 4-6, pp. 166-170, 2010.

[20] J. Huang, S. Li, and L. Sun, "The normalized Laplacians, degree-Kirchhoff index and the spanning trees of linear hexagonal chains," Discrete Applied Mathematics, vol. 207, pp. 67-79, 2016.

[21] Y. Yang and H. Zhang, "Kirchhoff index of linear hexagonal chains," International Journal of Quantum Chemistry, vol. 108, no. 3, pp. 503-512, 2008.

[22] K. C. Das, "On the Kirchhoff index of graphs," Zeitschrift für Naturforschung A, vol. 68, no. 8-9, pp. 531-538, 2013.

[23] H. Deng, "On the minimum Kirchhoff index of graphs with a given number of cut edges," MATCH Communications in Mathematical and in Computer Chemistry, vol. 63, pp. 171180, 2010.

[24] Q. Deng and H. Chen, "On the Kirchhoff index of the complement of a bipartite graph," Linear Algebra and Its Applications, vol. 439, no. 1, pp. 167-173, 2013.

[25] Q. Deng and H. Chen, "On extremal bipartite unicyclic graphs," Linear Algebra and Its Applications, vol. 444, pp. 89-99, 2014.

[26] L. Feng, G. Yu, K. Xu, and Z. Jiang, "A note on the Kirchhoff index of bicyclic graphs," Ars Combinatoria, vol. 114, pp. 33-40, 2014.

[27] X. Gao, Y. Luo, and W. Liu, "Resistance distances and the Kirchhoff index in Cayley graphs," Discrete Applied Mathematics, vol. 159, no. 17, pp. 2050-2057, 2011.

[28] I. Gutman and B. Mohar, "The quasi-Wiener and the Kirchhoff indices coincide," Journal of Chemical Information and Computer Sciences, vol. 36, no. 5, pp. 982-985, 1996.

[29] J. Huang, S. Li, and Q. Zhao, "On extremal bipartite bicyclic graphs," Journal of Mathematical Analysis and Applications, vol. 436, no. 2, pp. 1242-1255, 2016.

[30] J. Huang, S. Li, and X. Li, “The normalized Laplacian, degreeKirchhoff index and spanning trees of the linear polyomino 
chains," Applied Mathematics and Computation, vol. 289, pp. 324-334, 2016.

[31] R. Li, "Lower bounds for the Kirchhoff index," MATCH Communications in Mathematical and in Computer Chemistry, vol. 70, pp. 163-174, 2013.

[32] J.-B. Liu, X.-F. Pan, L. Yu, and D. Li, "Complete characterization of bicyclic graphs with minimal Kirchhoff index," Discrete Applied Mathematics, vol. 200, pp. 95-107, 2016.

[33] J. L. Palacios, "On the Kirchhoff index of graphs with diameter 2," Discrete Applied Mathematics, vol. 184, pp. 196-201, 2015.

[34] S. Pirzada, H. Ganie, and I. Gutman, "Comparison between Laplacian-energy-like invariant and Kirchhoff index," The Electronic Journal of Linear Algebra, vol. 31, pp. 27-41, 2016.

[35] H. Wang, H. Hua, and D. Wang, "Cacti with minimum, second-minimum, and third-minimum Kirchhoff indices," Mathematical Communications, vol. 15, pp. 347-358, 2010.

[36] Y. Wang and W. Zhang, "Kirchhoff index of linear pentagonal chains," International Journal of Quantum Chemistry, vol. 110, no. 9, pp. 1594-1604, 2010.

[37] K. Xu, H. Liu, and K. C. Das, "The Kirchhoff index of quasitree graphs," Zeitschrift für Naturforschung A, vol. 70, no. 3, pp. 135-139, 2015.

[38] K. Xu, K. C. Das, and X.-D. Zhang, "Ordering connected graphs by their Kirchhoff indices," International Journal of Computer Mathematics, vol. 93, no. 10, pp. 1741-1755, 2016.

[39] Y. Yang and D. J. Klein, "Comparison theorems on resistance distances and Kirchhoff indices of S,T-isomers," Discrete Applied Mathematics, vol. 175, pp. 87-93, 2014.

[40] H. Zhang and Y. Yang, "Resistance distance and Kirchhoff index in circulant graphs," International Journal of Quantum Chemistry, vol. 107, no. 2, pp. 330-339, 2007.

[41] H. Zhang, Y. Yang, and C. Li, "Kirchhoff index of composite graphs," Discrete Applied Mathematics, vol. 157, no. 13, pp. 2918-2927, 2009.

[42] G. Huang, M. Kuang, and H. Deng, "The expected values of Kirchhoff indices in the random polyphenyl and spiro chains," Ars Mathematica Contemporanea, vol. 9, pp. 197207, 2015.

[43] H. Wang, J. Qin, and I. Gutman, "Wiener numbers of random pentagonal chains," Iranian Journal of Mathematical Chemistry, vol. 4, no. 1, pp. 59-76, 2013.

[44] S. Wei and X. Ke, "Wiener and Kirchhoff indices in random generalized polyomino chains," Ars Combinatoria, vol. 143, pp. 337-350, 2019.

[45] C. Xiao, H. Chen, and A. M. Raigorodskii, "A connection between the Kekule structures of pentagonal chains and the Hosoya index of caterpillar trees," Discrete Applied Mathematics, vol. 232, pp. 230-234, 2017.

[46] A. Ali, Z. Raza, and A. A. Bhatti, "Extremal pentagonal chains with respect to bond incident degree indices," Canadian Journal of Chemistry, vol. 94, no. 10, pp. 870-876, 2016.

[47] I. Gutman and T. Körtvélyesi, "Wiener indices and molecular surfaces," Zeitschrift für Naturforschung A, vol. 50, no. 7, pp. 669-671, 1995.

[48] I. Gutman, J. W. Kennedy, and L. V. Quintas, "Wiener numbers of random benzenoid chains," Chemical Physics Letters, vol. 173, no. 4, pp. 403-408, 1990.

[49] A. Chen and F. Zhang, "Wiener index and perfect matchings in random phenylene chains," MATCH Communications in Mathematical and in Computer Chemistry, vol. 61, pp. 623630, 2009.

[50] S. Wei, X. Ke, and Y. Wang, "Wiener indices in random cyclooctane chains," Wuhan University Journal of Natural Sciences, vol. 23, no. 6, pp. 498-502, 2018.
[51] S. Wei and W. C. Shiu, "Enumeration of Wiener indices in random polygonal chains," Journal of Mathematical Analysis and Applications, vol. 469, no. 2, pp. 537-548, 2019.

[52] I. Gutman, "The number of perfect matchings in a random hexagonal chain," Graph Theory Notes New York, vol. 16, pp. 26-28, 1989.

[53] I. Gutman, J. W. Kennedy, and L. V. Quintas, "Perfect matchings in random hexagonal chain graphs," Journal of Mathematical Chemistry, vol. 6, no. 1, pp. 377-383, 1991.

[54] G. Huang, M. Kuang, and H. Deng, "The expected values of Hosoya index and Merrifield-Simmons index in a random polyphenylene chain," Journal of Combinatorial Optimization, vol. 32, no. 2, pp. 550-562, 2016.

[55] C. Xiao, H. Chen, and L. Liu, "Perfect matchings in random pentagonal chains," Journal of Mathematical Chemistry, vol. 55, no. 9, pp. 1878-1886, 2017. 